\title{
Avaliação do pé nos portadores de diabetes melitus
}

RESUMO | Objetivo: Avaliar o pé dos indivíduos portadores de Diabetes Melitus atendidos na atenção básica de um município do interior paulista. Método: Trata-se de um estudo quantitativo. Foram examinados 100 pés dos portadores de diabetes, no período de 1 a 31 de julho de 2019, aos usuários dos serviços de saúde do município (Estratégia Saúde da Família (ESF) e Unidade Básicas de Saúde (UBS)). Resultados: Houve predominância do sexo feminino, 56\% declararam que continham ensino fundamental incompleto. A hipertensão arterial foi a comorbidade mais citada. Na avaliação da história clinica associada aos cuidados com os pés, $87 \%$ dos entrevistados, declararam que nunca tiveram nenhum tipo de ulceração nos pés. Na inspeção e avaliação dos pés dos entrevistados, constatou-se que $94 \%$ não tinham nenhum tipo de lesões nos pés. Conclusão: 0 estudo reforçou a importância da participação dos profissionais de saúde atuando na prevenção do pé diabético.

Palavras-chaves: Pé diabético; Diabetes mellitus; Autocuidado com os pés.

ABSTRACT | Objective: To evaluate the foot of individuals with Diabetes Melitus assisted in primary care in a city in the interior of São Paulo. Method: This is a quantitative study. One hundred feet of people with diabetes were examined, between the 1st and 31st of July, 2019, users of health services in the municipality (Family Health Strategy (ESF) and Basic Health Units (UBS). Results: There was a predominance of females, $56 \%$ declared that they had incomplete primary education. Hypertension was the most cited comorbidity. In the evaluation of the clinical history associated with foot care, $87 \%$ of the interviewees stated that they had never had any type of foot ulceration. Upon inspection and assessment of the respondents' feet, it was found that $94 \%$ did not have any type of foot injury. Conclusion: The study reinforced the importance of the participation of health professionals working in the prevention of diabetic foot. Keywords: Diabetic foot; Diabetes mellitus; Self-care of the feet.

RESUMEN | Objetivo: Evaluar el pie de personas con Diabetes Melitus atendidas en atención primaria en una ciudad del interior de São Paulo. Método: Este es un estudio cuantitativo. Se examinaron cien pies de personas con diabetes, del 1 al 31 de julio de 2019, usuarios de los servicios de salud del municipio (Estrategia Salud de la Familia (ESF) y Unidades Básicas de Salud (UBS). Resultados: Predominó el sexo femenino, el 56\% declaró tener educación primaria incompleta. La hipertensión fue la comorbilidad más citada. En la evaluación de la historia clínica asociada al cuidado de los pies, el 87\% de los entrevistados afirmó no haber tenido nunca ningún tipo de ulceración del pie. Tras la inspección y evaluación de los pies de los encuestados, se encontró que el 94\% no tenía ningún tipo de lesión en el pie. Conclusión: El estudio reforzó la importancia de la participación de los profesionales sanitarios que trabajan en la prevención del pie diabético.

Palabras claves: Pie diabético; Complicaciones de la diabetes; Autocuidado.

\section{Allison Vinicius Bernardo}

Discente da Graduação em Enfermagem, Centro Universitário de Lins - UNILINS. Lins (SP), Brasil.

ORCID: 0000-0002-5636-3711

\section{Caroline Lima Noronha Lô}

Discente da Graduação em Enfermagem, Centro Universitário de Lins - UNILINS. Lins (SP), Brasil.

ORCID: 0000-0002-9408-7420

\section{Fabio Renato Lombardi}

Biólogo. Professor. Graduação em Ciências Biológicas, Centro Universitário de Lins - UNILINS - Lins (SP), Brasil. Doutor em Biofísica Molecar, pela Universidade Estadual Paulista (UNESP), campus de São José do Rio Preto. ORCID: 0000-0002-3610-4528

\section{Sabrina Piccineli Zanchettin Silva}

Enfermeira. Professora, Graduação em Enfermagem, Centro universitário de Lins - UNILINS. Lins (SP), Brasil. Mestre em biologia e envelhecimento pela Faculdade de Medicina de Marilia - FAMEMA.

ORCID: 0000-0002- 5763-6814

\section{Recebido em: 08/04/2021}

Aprovado em: 17/05/2021

\section{INTRODUÇ̃̃O}

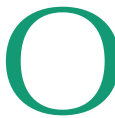
Diabetes mellitus caracteriza-se por uma síndrome do metabolismo de carboidratos, lipídeos e proteínas causada, tanto pela ausência de secreção de insulina quanto pela diminuição da sensibilidade dos tecidos à insulina ${ }^{(1)}$. Pode ser classificada em 2 tipos: tipo 1, ou dependente de insulina causado pela ausência de secreção da insulina; e o tipo 2, também chamado de diabetes mellitus não dependente de insulina (DMNID), causado pela diminuição da sensibilidade dos tecidos-alvo ao efeito metabólico da insulina ${ }^{(2)}$.

Acarreta diversas consequências a vida do portador, dentre elas, o pé diabético ou neuropatia. Essa complicação pode causar formigamento, dor, infecção, ulceração. Na neuropatia diabética peri- férica, todas as fibras, sensitivas, motoras, autonômicas pode haver redução de sensibilidade devido aos danos que a falta de controle da glicose causa aos nervos. Essa condição torna-se uma vulnerabilidade à ulceras e infecções que podem culminar em amputação(3).

As amputações representam um dos mais devastadores problemas associados à doença pois provoca grande impacto na vida pessoal, no âmbito familiar e socioeconômico, além do comprometimento da autoimagem e perda da capacidade produtiva, contextualizando a importância da implantação de medidas preventivas dessa complicação nos portadores de diabetes ${ }^{(4)}$.

\section{MÉTODO}

Trata-se de um estudo quantitativo, realizado no município de Lins, situado na região centro-oeste do estado de São Paulo. Foram examinados 100 pés dos portadores de diabetes, no período de 1 
a 31 de julho de 2019, aos usuários dos serviços de saúde do município (Estraté-

gia Saúde da Família (ESF) e Unidade Básicas de Saúde (UBS)).

\section{Tabela 1 - Caracterização sociodemográfica dos pacientes}

\section{Faixa etária}

30 a 39 anos

40 a 49 anos

50 a 59 anos

60 a 69 anos

$>70$ anos

Estado civil

Solteiro (a)

Casado (a)

Divorciado/separado (a)

Amasiado (a)

Viúvo (a)

Escolaridade

Analfabeto

Ens. fund. incompleto

Ens. fund. completo

Ens. médio incompleto

Ens. médio completo

Ens. sup. incompleto

Ens. sup. completo

Comorbidades

Hipertensão

Dislipidemia

Depressão

Alzheimer

Outras

Fonte: Elaborado pelo autor.

Tabela 2 - Caracterização dos cuidados com os pés, Lins - 2020.

\section{Variáveis}

Já apresentou algum tipo de ulceração nos pés?

Apresenta dor, principalmente noturna que melhora quando caminha?

Existe fraqueza muscular nos pés ou MMII?

Apresenta pontadas, agulhadas, formigamentos, nos MMII?

Apresenta dor ao caminhar?

Tem hábito de caminhar descalço?

Tem apoio da família em relação a saúde?

É tabagista?

Fonte: Elaborada pelo autor
A amostra total era composta de 1849 indivíduos, através do cálculo amostral considerando margem de erro de $10 \%$ e confiabilidade de $95 \%$, resultando numa amostra estimada de 92 indivíduos.

Foram inclusos na pesquisa todos os usuários dos serviços municipais de saúde, maiores de 18 anos e portadores de diabetes com diagnóstico.

O estudo contou com instrumento de caracterização sociodemográfica e de avaliação e inspeção dos membros inferiores. Assim, após consentimento positivo em participar da pesquisa e assinar o Termo de Consentimento Livre e Esclarecido (TCLE), o entrevistado foi submetido a avaliação. O estudo foi aprovado pelo Comitê de Ética em Pesquisa da Universidade de Marília - UNIMAR - Faculdade de Medicina de Enfermagem, sob $\mathrm{n}^{\circ}$ CAAE: 09546619.9.0000.5496.

Cabe destacar que quando detectada possíveis alterações neuropáticas, o entrevistado foi orientado e também comunicou-se a unidade de referência.

Os dados foram analisados através do Excel 2010 e organizados em tabelas.

\section{RESULTADOS}

Entre os 100 pacientes diabéticos que constituíram a amostra, houve predominância do sexo feminino apresentando $75 \%$ da amostragem, a faixa etária média foi de pacientes acima de 70 anos. Dos entrevistados, 56\% declararam que continham ensino fundamental incompleto. A hipertensão arterial foi a comorbidade mais citada, $83 \%$ dos entrevistados, conforme evidenciado na Tabela 1.

$\mathrm{Na}$ avaliação da história clinica associada aos cuidados com os pés, $87 \%$ dos entrevistados, declararam que nunca tiveram nenhum tipo de ulceração nos pés, enquanto que $86 \%$ disseram não ter o hábito de caminhar descalço, $52 \%$ declararam sentir pontadas, aguIhas e formigamentos nos pés, $42 \%$ relataram presença de dor nos pés ao caminhar, $10 \%$ não tem apoio da família em relação a saúde e 90\% declararam 
não ser tabagista, conforme pode ser visto na Tabela 2.

Apenas 14\% dos entrevistados já receberam orientações relacionadas ao cuidado com os pés e $26 \%$ já tiveram seus pés examinados por algum profissional. Na inspeção e avaliação dos pés dos entrevistados, constatou-se que 94\% não tinham nenhum tipo de lesões nos pés; $96 \%$ dos pacientes declaram usar sapatos adequados, ou seja, nenhum tipo de calçado que aperte os pés ou que causam lesões.

$\mathrm{Na}$ avaliação de sensibilidade, constatou-se que $88 \%$ dos entrevistados, teve avaliação satisfatória no pé direito e $86 \%$ no pé esquerdo. Quanto aos testes vasculares, no pé direito foi observado a presença do pulso tibial posterior em $77 \%$ dos pacientes. Já o pulso pedioso $77 \%$ dos pacientes tiveram o pulso presente. No pé esquerdo, foi observado a presença do pulso tibial posterior em $75 \%$ dos pacientes, no pulso pedioso $77 \%$ dos pacientes tiveram o pulso presente.

\section{DISCUSSÃO}

O perfil traçado na atual pesquisa corrobora com o demonstrado em outros estudos ${ }^{(5-7)}$, destacando que a baixa escolaridade, tem influência no cuidado dos pés, haja vista que essas pessoas apresentam dificuldades de acesso às informações, implicando em risco diferenciados no adoecer e morrer ${ }^{(8,9)}$.

A hipertensão arterial relatada pelos participantes, confirma o cenário de outras regiões ${ }^{(10)}$, na qual ela é citada como a comorbidade mais frequente. Nesse contexto, vê-se a importância da instrução na mudança da alimentação, o uso correto da medicação e práticas de exercício físico, como a caminhada.

Em relação ao histórico do paciente 87\% não apresentou algum tipo de ulceração nos pés, embora, ainda se faz necessário manter as medidas de prevenção e cuidados para evitar o aparecimento das úlceras ${ }^{(11)}$. Em um estudo realizado em Minas Gerais ${ }^{(22)}$ apenas 5,7\% dos participantes apresentaram ulcerações nos pés, enquanto em nosso estudo 13\% dos participantes relataram apresentar ou ter apresentado ulceras.

Dos sintomas sensoriais avaliados $58 \%$ não apresentaram dor ao caminhar, e $42 \%$ se queixaram de dores, ainda que o percentual seja menor a intensidade da dor varia de moderada a grave ${ }^{(12)}$ apontando para os outros sintomas analisados como pontadas, agulhadas e formigamentos que obtiveram $52 \%$ de confirmação. Frente as medidas de prevenção com os pés diabéticos, enfatizamos a importância e a necessidade de se realizar o teste de sensibilidade nos pés dos pacientes com diabetes mellitus.

A participação da família na vida do portador de diabetes mellitus é de grande importância, para melhor adequação dos cuidados com o portador e a doença, aumentando a prevenção e o retardamento de possíveis complicações decorrentes dela. No presente estudo $90 \%$ dos participantes relatam obter o apoio da família e 10\% afirmam não possuir. O ato de cuidar é uma responsabilidade difícil, acompanhar e conviver com o adoecimento provoca reações negativas o que provoca mudanças na dinâmica familiar, o que pode gerar uma desqualificação no cuidar ${ }^{(14)}$, ou seja, cabe a enfermagem fornecer as informações sobre a doença e os cuidados, mirando em uma estratégia que vise o entendimento e evolução desses pacientes ${ }^{(15)}$.

O uso do tabaco é um dos fatores que formigamentos que obtiveram $52 \%$ de confirmação. dificultam a cicatrização e piora as lesões da pele associadas à diabetes. ${ }^{(16)} \mathrm{Em}$ nossa pesquisa $90 \%$ declarou não ser fumante, semelhante aos dados coletados de uma pesquisa em Fortaleza onde $76 \%{ }^{(7)}$ dos participantes também não possui este hábito, demonstrando uma diminuição no uso do tabaco. Devido aos problemas que o tabaco acarreta a vida do fumante é importante fornecer informações sobre os malefícios que este vicio pode causar em sua saúde.

No que se refere ao cuidado com os pés $86 \%$ dos participantes não receberam 
orientação das formas de cuidado pelos profissionais de saúde. Essa ausência de orientações a esses pacientes ocasiona em complicações graves e sérias. Um estudo realizado no interior paulista comprovou que a equipe de saúde é responsável por expandir o conhecimento sobre o cuidado com os pés ${ }^{(10)}$.

A inspeção dos pés diabéticos deve ser feita diariamente para verificar presença de calosidade, fissura e pele ressecada, podendo este ser realizado por profissional da saúde, por algum familiar e até mesmo o próprio portador. $\mathrm{Na}$ atual pesquisa $74 \%$ dos participantes declararam não terem os pés examinados por algum profissional de saúde, e no estudo realizado no Triângulo Mineiro 94\% afirmaram não realizar exame dos pés por profissionais de saúde 1 vez ao ano ${ }^{(17)}$. A inspeção deve ser feita pelo enfermeiro para detectar presença de edema, verificar o corte das unhas, úlceras, tipo de calçado, sensibilidade, formato dos pés, quais cremes ou pomadas está sendo utilizado, para evitar agravamento das complicações, com a função de prevenir e ensinar.

Quanto a inspeção dos pés e calçados 94\% não apresentaram alterações em seus pés, dados evolutivos ao estudo feito em Minas Gerais onde 52\% não apresentavam nenhum tipo de deformidade em seus pés. ${ }^{(18)}$ Podemos assim estimar como um aumento no cuidado da prevenção de ulceração. Em um estudo realizado no Paraná ${ }^{(19)} 60 \%$ dos participantes utilizavam calçados inadequados, enquanto em nossa pesquisa 96\% apresentavam o uso adequado dos calçados, constituindo um elemento que se otimizou devido ao acréscimo de informações sobre o uso correto de calçados.

Os testes neurológicos realizados nos trouxeram os seguintes dados: $12 \%$ dos pacientes não sentiam a pressão do monofilamento no pé direito, e no pé esquerdo $14 \%$ não sentiam a essa pressão, num estudo realizado em Belo Horizonte com portadores de diabetes mellitus $62,50 \%$ (18) não teve sensibilidade em ambos os pés. Observa-se que essa diferença aponta para o avanço que obtivemos na prevenção durante esses 8 anos, onde $88 \%$ sentiram a pressão do monofilamento no pé direito e $86 \%$ no pé esquerdo.

Com a realização dos testes vasculares no pulso pedioso notamos que no pé

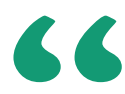

direito e esquerdo $77 \%$ dos participantes tinham o pulso presente, contraditório a outro estudo realizado no Piauí onde $40,7 \%$ foi presente no pé direito e 39,4\% no pé esquerdo ${ }^{(20)}$.

Quanto a ausência do pulso pedioso, no pé direito $11 \%$ foi ausente e no pé esquerdo apenas $8 \%$, resultados melhores aos encontrados a outra pesquisa de Minas Gerais onde 41,6\% foi ausente. ${ }^{(22)}$ Os dados obtidos com a palpação do pulso tibial posterior foram semelhantes ao pedioso, no qual $77 \%$ tiveram o pulso presente no pé direito e $75 \%$ presente no pé esquerdo, em um estudo de Fortaleza $89 \%$ foi presente no pé direito e $88 \%$ foi presente no esquerdo ${ }^{(7)}$. O pulso tibial posterior teve $13 \%$ de ausência para ambos os pés, enquanto no estudo de Belo Horizonte apenas $9,2 \%$ foi ausente. ${ }^{(20)}$ Os pulsos tibiais e o pedioso, podem estar ausentes sem que o indivíduo tenha qualquer déficit de suprimento sanguíneo nos membros. Portanto, essa característica deve ser analisada na ausência de palpação ${ }^{(23)}$.

\section{CONCLUSÃO}

Com base em nosso estudo, foi observado um baixo grau de escolaridade pela maioria dos pacientes por não terem concluído o ensino fundamental, e devido a esse fator há uma grande perda de informação referente aos cuidados com os pés. Além da falha na comunicação e orientação partindo dos profissionais de saúde, pois obtivemos uma grande quantidade de participantes que declararam nunca terem recebido orientações de cuidados com os pés.

Conforme os dados coletados ressaltamos a importância da participação dos profissionais de saúde atuando na prevenção do pé diabético e manutenção da comunicação. Diante desse cenário sugerimos algumas medidas preventivas para os profissionais perante o portador de diabetes, como: visitas domiciliares mensais para avaliação e tratamento dos pés diabéticos, acompanhamento da 
glicemia capilar e orientações quanto a doença, distribuição de óleos ou cremes hidratantes, oferecer panfletos informativos sobre a diabetes e quanto aos cuidados necessários e realizar treinamentos e capacitações com sua equipe, para enfatizar a necessidade de orientação aos pacientes portadores de diabetes mellitus.

Por fim, lembramos a importância da criação de grupos de caminhada, visto que aumentam a prevenção de doenças, estimula o convívio entre os participantes aumentando assim a interação social, ajuda no equilíbrio do corpo e no emagrecimento.

\section{Referências}

1. GUYTON, A.C; HALL, J.E.: Tratado de fisiologia médica. RIO DE JANEIRO. [livro]. Elsevier, 2006.

2. GUYTON, A.C; HALL, J.E.: Fisiologia humana e mecanismos das doenças. RIO DE JANEIRO [livro] Saunders, 1996.

3. Sociedade Brasileira de Diabetes [homepage na internet]. Complicações do Diabetes [acesso em 26 de julho de 2020]. Disponível em: https://www.diabetes.org.br/publico/complicacoes/complicacoes-do-diabetes.

4. Mello R, Pires $M$, Kede J. Ficha de avaliação clínica de membros inferiores para prevenção do pé diabético Clinical evaluation form of lower members for diabetic foot prevention. Revista de Pesquisa: Cuidado é Fundamental Online [Internet]. 2017 Jul 11; [Citado em 2020 Jun 30]; 9(3): 899-913. Disponível em: http://www.seer.unirio.br/index.php/cuidadofundamental/article/view/5468. 5. Boell JEW, Ribeiro RM, Silva DMGV da. Fatores de risco para o desencadeamento do pé diabético. Rev. Eletr. Enf. [Internet]. $30^{\circ}$ de junho de 2014 [citado $30^{\circ}$ de junho de 2020];16(2):386-93. Disponível em: https://www.revistas.ufg. br/fen/article/view/20460.

6- Ochoa-Vigo Kattia, Torquato Maria Teresa da Costa Gonçalves, Silvério Izilda Aparecida de Souza, Queiroz Flávia Alline de, De-La-Torre-Ugarte-Guanilo Mónica Cecilia, Pace Ana Emilia. Caracterização de pessoas com diabetes em unidades de atenção primária e secundária em relação a fatores desencadeantes do pé diabético. Acta paul. enferm. [Internet]. 2006. Sep [cited 2020 June 30]; 19(3):296-303. Available from: http://www.scielo.br/scielo.php?script=sci_arttext\&pid=S0103-21002006000300007\&lng=en.

7. Araujo, M. M. Alencar, A. M. P. G. Pés de risco para o desenvolvimento de ulcerações e amputações em diabéticos. Rev. Rene. Fortaleza. [internet]. 2009 [citado 23 de agosto de 2019]; 10(2):19-28. Disponível em: http://www.periodicos.ufc.br/rene/article/download/4760/3528.

8. Pace Ana Emília, Foss Milton Cesar, Ochoa-Vigo Kattia, Hayashida Miyeko. Fatores de risco para complicações em extremidades inferiores de pessoas com diabetes mellitus. Rev. bras. enferm. [Internet]. 2002 Oct [cited 2020 July 21] ; 55( 5 ): 514-521. Available from: http://www.scielo.br/scielo.php?script=sci_arttext\&pid=S0034-71672002000500006\&lng=en. https://doi. org/10.5935/0034-7167.20020067.

9. Flor Luisa Sorio, Campos Monica Rodrigues. Prevalência de diabetes mellitus e fatores associados na população adulta brasileira: evidências de um inquérito de base populacional. Rev. bras. epidemiol. [Internet]. 2017 Mar [cited 2020 July 21] ; 20( 1 ): 16-29. Available from: http://www.scielo.br/scielo. php?script=sci_arttext\&pid=S1415-790X2017000100016\&lng=en. https:// doi.org/10.1590/1980-5497201700010002.

10. Rocha Roseanne Montargil, Zanetti Maria Lúcia, Santos Manoel Antônio dos. Comportamento e conhecimento: fundamentos para prevenção do pé diabético. Acta paul. enferm. [Internet]. 2009 Feb [cited 2020 July 21] ; 22( 1 ): 17-23. Available from: http://www.scielo.br/scielo.php?script=sci_arttext\&pid=S0103-21002009000100003\&lng=en. https://doi.org/10.1590/S010321002009000100003.

11. Cubas Marcia Regina, Santos Odette Moura dos, Retzlaff Elis Marina Andrade, Telma Helouíse Letícia Cristiano, Andrade Iria Priscila Silva de, Moser Auristela D. de Lima et al . Pé diabético: orientações e conhecimento sobre cuidados preventivos. Fisioter. mov. [Internet]. 2013 Sep [cited 2020 July 21]; 26( 3 ): 647-655. Available from: http://www.scielo.br/scielo.php?script=sci_ arttext\&pid=S0103-51502013000300019\&lng=en. https://doi.org/10.1590/ S0103-51502013000300019.

12. Nascimento, Rayssa Tuana Lourenço, Lopes Cristiane Barbieri, Cotta Danielli Da Silva, Alencar Nayara Luiza Oliveira Rocha de, Valadão Analina Furtado, Motta Patrícia Gonçalves da. Neuropatia Diabética Dolorosa - aspectos clínicos, diagnóstico e tratamento: uma revisão de literatura. Revista Uningá, [internet]. 2015. [cited 2020 July 21]; 43(1). Disponível em: http://revista. uninga.br/index.php/uninga/article/view/1215.

13. Santos Michele Marchetti dos, Soethe Michely da Silva, Soares Antonio Vinicius. Avaliação da polineuropatia diabética e a prevenção de úlceras em idosos. Revista Kairós: Gerontologia. [internet]. 2018 [cited 2020 July 21]; 21(1): 355-376. Available from: https://revistas.pucsp.br/kairos/article/view/39888 https://doi.org/10.23925/2176-901X.2018v21i1p355-376.

14. Martins Maísa Mônica Flores, Rodrigues Maina Lima. Diabetes: adesão do paciente e o papel da família nessa nova realidade. Rev. Aten. Saúde [internet]. 2019. [cited 2020 July 26]; 17(59): 95-102. Availabre from: https://seer uscs.edu.br/index.php/revista_ciencias_saude/article/view/5838. https://doi. org/10.13037/ras.vol17n59.5838.

15. Pimentel Tayná Santos, Marques Dayse Rosângela Santos. Atuação do enfermeiro no controle da neuropatia periférica em pacientes portadores de diabetes mellitus tipo 2. Periodic. Set. [internet]. 2019. [cited 2020 July 21]; 5(2): 213-228. Available from: https://periodicos.set.edu.br/index.php/cadernobiologicas/article/view/6626.

16. Nunes E. Consumo de tabaco. Efeitos na saúde Revista Portuguesa de Medicina Geral e Familiar [série na Internet]. 2006 março 1; [Citado 2020 Julho 21]; 22(2). Disponivel em: https://www.rpmgf.pt/ojs/index.php/rpmgf/article/ view/10231.

17. Silva Pollyane Liliane, Rezende Marina Pereira, Ferreira Lúcia Aparecida, Dias Flavia Aparecida, Helmo Fernanda Rodrigues, Silveira Fabíola Cristina Oliveira. Cuidados de los pies: el conocimiento de las personas con diabetes mellitus inscritos en el programa de salud familiar. Enferm. glob. [Internet]. 2015. [cited 2020 July 21]; 14( 37 ):38-51. Disponible en: http://scielo.isciii. es/scielo.php?script=sci_arttext\&pid=\$1695-61412015000100003\&lng=es. 18. Guimarães, J. P. C. Classificação de risco para pé diabético em pessoas idosas com diabetes mellitus tipo 2. Belo Horizonte. Dissertação[Mestrado em Enfermagem] - Universidade Federal de Minas Gerais; 2011.

19. Audi Eduardo Godoi, Moreira Ricardo Castanho, Moreira Ana Cândida Martins Grossi, Pinheiro Eliana de Fátima Catussi, Mantovani Maria de Fátima, Araújo Arlinda Gonçalves de. Avaliação dos pés e classificação do risco para pé diabético: contribuições da enfermagem. Rev. Cog. Enf. [internet]. 2011. [cited 2020 July 26]; 16(2):240-6. Available from: https://revistas.ufpr.br/cogitare/ article/view/19975. http://dx.doi.org/10.5380/ce.v16i2.19975.

20. Vinicius Saura Cardoso, Alessandra Tanuri Magalhães, Baldomero Antônio Kato da Silva, Cristiano Sales da Silva, Dandara Beatriz Costa Gomes, Jefferson Carlos Araujo Silva. Avaliação funcional dos pés de portadores de diabetes tipo II. Rev Bras Promoç Saúde. [internet]. 2013. [cited 2020 July 26]; 26(4): 563-570. Available from: https://periodicos.unifor.br/RBPS/article/view/2329. https://doi.org/10.5020/2329.

21. MELLO, Rodrigo da Fontoura de Albuquerque; PIRES, Maria Lúcia Elias; $\mathrm{KEDE}$, Jorge. Ficha de avaliação clínica de membros inferiores para prevenção do pé diabético Clinical evaluation form of lower members for diabetic foot prevention. Revista de Pesquisa: Cuidado é Fundamental Online, [S.I.], v. $9, \mathrm{n}$. 3, p. 899-913, july 2017. ISSN 2175-5361. Disponível em: <http://www.seer. unirio.br/index.php/cuidadofundamental/article/view/5468>. Acesso em: 26 july 2020. doi:http://dx.doi.org/10.9789/2175-5361.rpcfo.v9.5468.

22. Delfraro Carmo, T., Almeida, J., do Carmo, D., Godoi, M., Silva, M., \& Carmo, T. (2017). • Monofilamento de Semmes-Weinstein: uma avaliação da sensibilidade protetora dos pés na prevenção da úlcera plantar entre pacientes diabéticos. Ciência ET Praxis (Qualis B3 - 2017-2018) [internet], 8(15), 29-34. Recuperado de http://revista.uemg.br/index.php/praxys/article/view/2151 23. Porto, C. C. Exame clínico. Rio de Janeiro. [livro]. Guanabara Koogan, 1992. 